\title{
Lomboradiculalgie Du Sujet Âgé En Consultation Rhumatologique À Lomé, Togo
}

\author{
Kakpovi Kodjo \\ Service de Rhumatologie, CHU Sylvanus Olympio, Lomé, Togo \\ Fianyo Eyram \\ Service de Rhumatologie, CHR Lomé Commune, Lomé, Togo
}

Houzou Prenam

Service de Rhumatologie, CHU Kara, Kara, Togo

Sogan Elias

Service de Rhumatologie, CHU Sylvanus Olympio, Lomé, Togo

Koffi-Tessio Viwalé Etonam Sika

Service de Rhumatologie, CHU Sylvanus Olympio, Lomé, Togo

Tagbor Komi Cyrille

Service de Rhumatologie, Hôpital de Bè, Lomé, Togo

\section{Oniankitan Owonayo}

Service de Rhumatologie, CHR Lomé Commune, Lomé, Togo

\section{Mijiyawa Moustafa}

Service de Rhumatologie, CHU Sylvanus Olympio, Lomé, Togo

Doi: 10.19044/esj.2017.v13n30p222 URL:http://dx.doi.org/10.19044/esj.2017.v13n30p222

Abstract

Objective: To study the epidemiological, diagnostic, therapeutic and outcome aspects of low back pain with radicular pain in rheumatology ward at Sylvanus Olympio Teaching Hospital at Lome, TOGO. Methods: It is a cross sectional study of 298 patients of 65 years and above, suffering from low back pain with radicular pain. They were admitted in the rheumatology ward of Sylvanus Olympio Teaching Hospital from January 2010 to December 2015. Results: 298 out of 768 patients examined within a period of five years $(38.80 \%)$ were suffering from low back pain with radicular pain. These 298 patients comprises of 215 women $(72.15 \%)$ and 83 men $(27.85 \%)$ with an average age of 62.04 years at the onset. The mean age at the consultation was $70.72 \pm 5.5$ years. The mean duration of the diseases was $10.28 \pm 23.81$ months. The major diseases observed were degenerative disc (279 cases : 93.61\%), bone tumors (16 cases : $5.38 \%$ ), and spondylodiscitis $(03$ cases : $1.01 \%)$. The clinical manifestations of the degenerative disc of the lumbar spine were the LBP (209 cases : 74,91\%) 
and the narrowed lumbar canal (47 cases : 16.85\%). The bone tumors were dominated by prostate cancer (five cases : 12,02\%). The spondylodiscitis, probably of tuberculous origin, affected three patients, among whom was one Human Immunodeficiency Virus (HIV) patient. Conclusion: Degenerative disc is a frequent pathology nowadays, both in the Togolese population and in the elderly in particular. Nevertheless, efforts should be made to eliminate specific back pain in the elderly, whose clinical picture may be misleading

Keywords: Elderly, Low back pain, Radicular pain, Osteoarthritis, BoneTumor, Black Africa

\section{Resume}

Objectif: Étudier les aspects épidémiologiques, diagnostiques, thérapeutiques et évolutifs des lomboradiculalgies du sujet âgé en milieu rhumatologique au CHU Sylvanus Olympio de Lomé (TOGO). Méthodes: Il s'agit d'une étude transversale portant sur 298 dossiers de patients âgés de 65 ans et plus, souffrant de lomboradiculalgie, ayant été consultés ou hospitalisés dans le service de rhumatologie du CHU-Sylvanus Olympio entre janvier 2010 et décembre 2015. Résultats: 298 des 768 patients âgés vus en cinq ans (38,80\%) souffraient d'une lomboradiculalgie. Ces 298 patients qui se répartissaient en 215 femmes $(72,15 \%)$ et 83 hommes $(27,85$ $\%$ ) avaient en moyenne 62,04 ans au début des symptômes. L'âge moyen à la consultation était de 70,72 $\pm 5,5$ ans. La durée moyenne d'évolution était de 10,28 $\pm 23,81$ mois. L'atteinte dégénérative du rachis (279 cas : 93,61\%), les tumeurs vertébrales (16 cas : 5,36\%) et les spondylodiscites infectieuses (03 cas : 1,01\%) ont été les principales formes étiologiques observées. Le conflit disco-radiculaire (209 cas, 74,91\%) et le canal lombaire rétréci (47 cas, 16,85\%) constituaient les formes cliniques d'atteinte dégénérative du rachis lombaire. Les tumeurs étaient dominées par les métastases dont cinq cas $(12,02 \%)$ d'origine prostatique. La spondylodiscite d'origine probablement tuberculeuse a affecté 03 patientes dont une porteuse du virus de l'immunodéficience humaine.Conclusion: La lomboradiculalgie commune est une pathologie fréquente de nos jours, tant dans la population togolaise que chez le sujet âgé en particulier. Il faut néanmoins s'acharner à éliminer une lomboradiculalgie secondaire chez le sujet âgé, dont le tableau clinique peut être trompeur.

Mots-clés: Sujet âgé, Lomboradiculalgie, Arthrose, Tumeur osseuse, Afrique Noire

\section{Introduction}

Selon l'Organisation Mondiale de la Santé, le sujet âgé se définit comme toute personne ayant un âge supérieur ou égal à 65 ans (Longue vie 
et autonomie, 2016). La population de sujet âgé (65 ans et plus) dans le monde s'estimait à environ 668.672.959 personnes, soit $9 \%$ de la population mondiale en 2016 (Organisation Mondiale de la Santé, 2016).Cette population de sujet âgé est sujette à des affections aigues ou chroniques résultantes des facteurs auxquels elle a été soumise et du processus normal $\mathrm{du}$ vieillissement. Parmi ces pathologies, La pathologie rachidiennelombaire représente après la maladie cardio-vasculaire le $2^{\text {ème }}$ groupe d'affections fréquemment rencontrées chez le sujet âgé (Institut de Recherche et Documentation en Economie de Santé, 2013).Cette pathologie rachidienne lombaire est un problème de santé qui entraîne plus souvent chez les personnes âgées, un handicap et une altération importante de la qualité de vie (Wong et al., 2017). Dans les pays développés, la fréquence de la lomboradiculalgie du sujet âgé varie de $25 \%$ à $35 \%$ avec un âge moyen de 75 ans et une prédominance féminine (Cecchi et al., 2006; Jimenez-Sanchez et al., 2012). Les facteurs de risques des lomboradiculalgies chez le sujet âgé sont représentés par la pauvreté, la faible activité physique, les chutes et les syndromes dépressifs majeurs (Mehsra et al., 2017).En Afrique, la prévalence des lomboradiculalgies du sujet âgé varie de $20 \%$ à $50 \%$ avec un âge moyen autour de 70 ans (Haddada et al., 2005; Ouédraogo et al., 2011). $\mathrm{Au}$ Togo, dans une étude hospitalière en 2004, elle était de $18 \%$ dans une large population de rhumatisants dont l'âge moyen était de 49 ans (Oniankitan et al., 2008). Aucune étude des affections rhumatismales n'a été consacrée exclusivement au sujet âgé au Togo. Ce travail a pour but d'étudier les aspects épidémiologiques, cliniques, radiographiques, étiologiques, thérapeutiques et évolutifs des lomboradiculalgies du sujet âgé en milieu rhumatologique au Centre Hospitalier Universitaire Sylvanus Olympio (CHU SO) de Lomé.

\section{Patients et methodes}

Il s'est agi d'une étude rétrospective de 298 dossiers de patients âgés de 65 ans et plus souffrant de lomboradiculalgie, ayant consultés ou hospitalisés dans le service de rhumatologie du CHU-Sylvanus Olympio entre janvier 2010 et décembre 2015. Les patients ayant un dossier médical incomplet ou inexploitable, ou ceux présentant une affection ostéoarticulaire traumatique ou post traumatique n'ont pas été inclus dans l'étude. Les paramètres étudiés étaient les données démographiques (l'âge, le sexe), le motif de consultation, la durée moyenne d'évolution de la maladie, les antécédents, les signes généraux, les signes physiques et les résultats d'éventuels examens paracliniques réalisés en fonction de l'orientation diagnostique. L'imagerie par résonance magnétique et la scintigraphie n'ont été réalisées chez aucun patient. Le diagnostic des différentes formes cliniques d'atteinte dégénérative du rachis a reposé sur l'existence d'une 
douleur mécanique, la conservation de l'état général, l'absence de signes biologiques d'inflammation et l'existence des signes radiologiques. Les spondylodiscites infectieuses et les tumeurs osseuses ont été de diagnostic radioclinique. Le myélome multiple a été reconnu sur la base de l'électrophorèse des protides sériques (pic monoclonal) et du myélogramme (plasmocytes dystrophiques). L'obésité a été définie comme un index de masse corporelle supérieurà $30 \mathrm{~kg} / \mathrm{m}^{2}$.

\section{Resultats}

La lomboradiculalgie a motivé la consultation (257 patients) ou 1'hospitalisation (41 patients) de 298 des 768 sujets âgés $(38,80 \%)$ examinés en 6 ans. Ces 298 patients se répartissaient en 215 femmes $(72,15 \%)$ et 83 hommes $(27,85 \%)$ soit une sex-ratio de 2,59. Leur âge moyen à la consultation était de 70,72 $\pm 5,55$ ans avec des extrêmes de 65 ans et 87 ans. La tranche d'âge de 65 ans à 69 ans était la plus représentée dans 46,64\% (Tableau I).

Tableau I. Répartition des patients selon les tranches d'âge de 5 ans

\begin{tabular}{ccc}
\hline & Nombre (n) & Pourcentage (\%) \\
\hline$[65-69[$ & 139 & 46,64 \\
{$[70-74[$} & 93 & 31,21 \\
{$[75-79[$} & 32 & 10,74 \\
{$[80-84[$} & 28 & 09,40 \\
{$[85-89[$} & 06 & 02,01 \\
\hline Total & 298 & 100 \\
\hline
\end{tabular}

Les comorbidités étaient principalement l'hypertension artérielle dans $47,65 \%$ des cas et les gastropathies dans $11,41 \%$ des cas. Cent deux patients $(40,48 \%)$ étaient obèses. La durée moyenne entre le début des symptômes et la première consultation rhumatologique était de 10,28 mois avec des extrêmes de 1 mois à 240 mois. Le mode de début était progressif dans $84,56 \%$ des cas et brutal dans $16,44 \%$ des cas. La douleur était mécanique $(83,56 \%)$, inflammatoire $(10,40 \%)$ et mixte $(06,04 \%)$. L'irradiation aux membres inférieurs était unilatérale dans $80,35 \%$ des cas avec une prédominance de L5 dans 37,25\%. Le périmètre de marche était limité chez 176 patients $(59,06 \%)$ dont $140(79,55 \%)$ avait moins de 500 mètres.

L'atteinte dégénérative du rachis (279 cas; 93,61\%) a été la principale forme étiologique observée. La lomboradiculalgie par probable conflit disco-radiculaire a été la forme clinique la plus retrouvée dans $74,91 \%$ des cas (Tableau II).

Tableau II. données démographiques des 298 patients en fonction du diagnostic

\begin{tabular}{cccc} 
Sexe ratio & Age (ans) à la & Age (ans) en & Durée (mois) \\
$\mathrm{F} / \mathrm{H}^{* * *}$ & consultation & début des & d'évolution \\
& $(\mathrm{m} \pm \mathrm{ET})^{* * * *}$ & symptômes & des \\
& & $(\mathrm{m} \pm \mathrm{ET})$ & symptômes $)$ \\
\hline
\end{tabular}




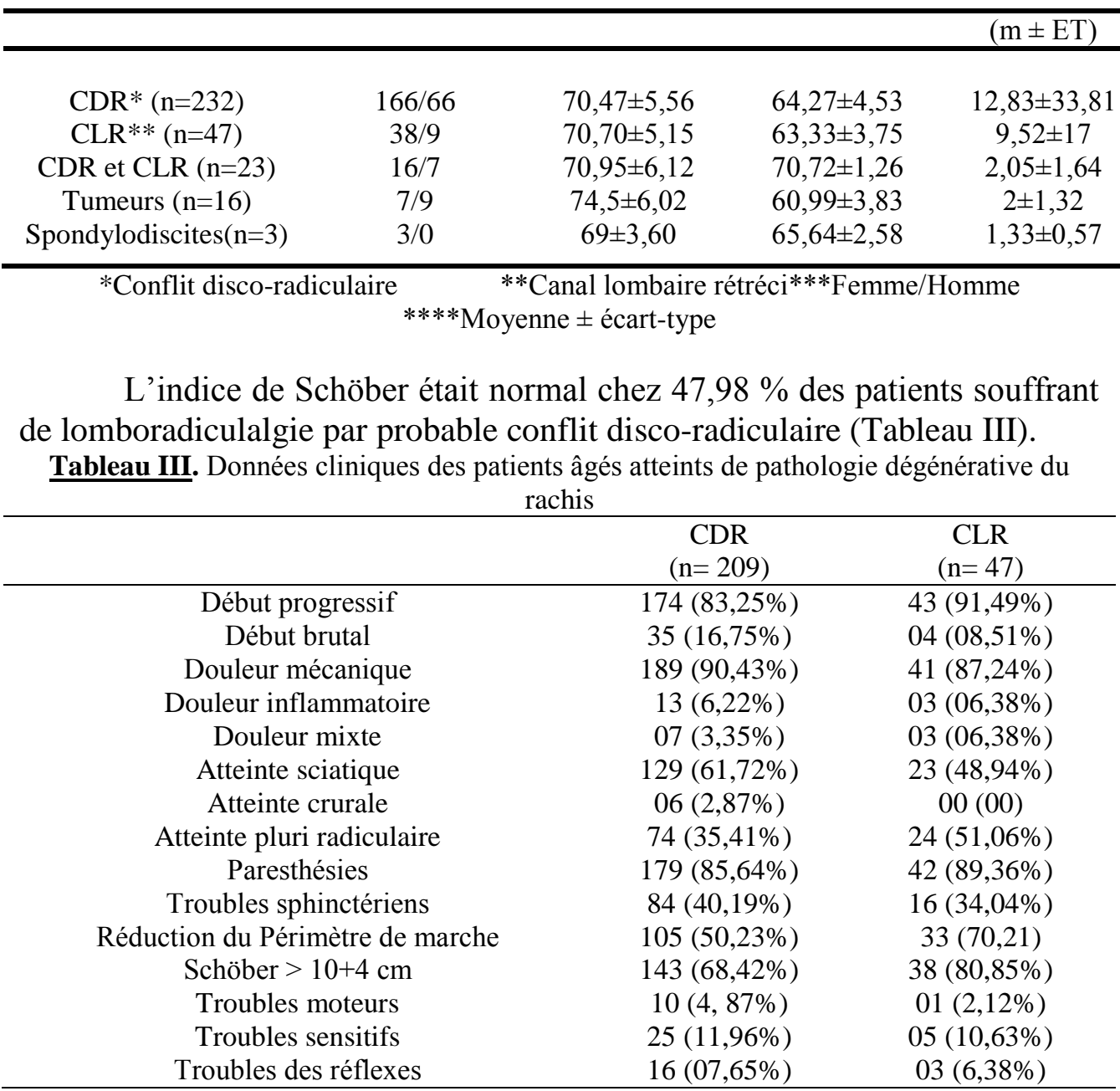

La discopathie dégénérative isolée $(71,14 \%)$, ou associée à un listhésis $(33,55 \%)$ ou à une arthrose interapophysaire postérieure (AIAP) $(02,68 \%)$ était la principale anomalie radiographique en cas d'atteinte dégénérative. La tomodensitométrie (TDM) réalisée seulement chez 22 patients $(07,38 \%)$ a objectivé un canal lombaire rétréci (CLR) $(14 ; 63,63 \%)$, une AIAP $(10 ; 45,45 \%)$ un listhésis $(06 ; 27,22 \%)$, et une hernie discale $(01 ; 04,54 \%)$. Le traitement chirurgical proposé chez 24 patients n'a été réalisé que chez sept. Le traitement médical était constitué d'infiltrations épidurales $\quad(77,77 \%)$, d'antalgiques $\quad(66,31 \%)$, d'AINS $(25,81 \%)$, d'anxiolytiques $(22,58 \%)$ et de myorelaxants $(2,51 \%)$. L'évolution a été favorable dans $97,46 \%$ des cas.

Les tumeurs osseuses $(5,36 \%)$ étaient représentées par les tumeurs d'origine indéterminée $(2,34 \%)$, les métastases prostatiques $(1,67 \%)$, le 
myélome $(0,67 \%)$, la tumeur d'origine bronchique $(0,34 \%)$ et le lymphome lymphoïde chronique $(0,34 \%)$.

La spondylodiscite infectieuse était d'origine probablement tuberculeuse dans $1,01 \%$ des cas et ne touchait que les femmes. Une des trois patientes était porteuse du VIH.

\section{Discussion}

Durant 6 ans sur lesquelles a porté cette étude, la lomboradiculalgie a été le motif de consultation ou d'hospitalisation de $38,80 \%$ de sujet âgé vus dans le service de rhumatologie du CHU Sylvanus OLYMPIO. La pathologie dégénérative $(93,61 \%)$ a été la principale forme étiologique. Les lomboradiculalgies symptomatiques ont été plus souvent le fait de tumeurs $(5,36 \%)$ que de spondylodiscites infectieuses $(1,01 \%)$. En dépit de ses insuffisances rétrospectives (recrutement hospitalier, étroitesse du plateau technique), cette étude, comme celles effectuées dans d'autres pays, témoigne de l'importance de la pathologie dégénérative du rachis en Afrique chez le sujet âgé (Haddada et al., 2005; Ouédraogo et al., 2011); tout ceci lié à une meilleure espérance de vie. La prédominance féminine $(72,15 \%)$ observée dans notre étude s'explique parle le rôle majeur des femmes dans les travaux ménagers et l'hygiène quotidienne du domicile dans nos milieux africains, et par l'espérance de vie plus élevée chez les femmes $(67,17 \mathrm{ans}$ contre 61,91 ans chez les hommes) au Togo (Institut National de la Statistique et des Etudes Economiques et Démographiques, 2015) tout comme dans d'autres pays africains (Ouédraogo et al., 2011; Diomande et al., 2015). L'âge moyen de 70,72 ans était similaire aux données de la littérature (Sghir et al., 2011; Rahali-Khachlouf et al., 2009). Ceci est dû à aux différentes contraintes subies par le rachis et à la fragilité de l'organisme du sujet âgé.

Les caractères démographiques et sémiologiques de ces patients atteints de lomboradiculalgie commune étaient comparables aux données de la littérature (Sghir et al., 2011; Werndle et al., 2012; Rainville et al., 2013).

Le faible taux de réalisation de la TDM $(7,38 \%)$ associé au diagnostic présomptif expliquerait certainement le taux élevé de CDR et le taux faible de CLR dans notre étude contrairement aux données de la littérature (Haddada et al., 2005; Sghir et al., 2011; Cheour et al., 2007). Le caractère peu déficitaire de la lombosciatique et le manque de moyens financiers expliquent la rareté de la tomodensitométrie rachidienne dans notre série. Cela reflète une prescription rationnelle de la TDM en raison de son intérêt surtout chirurgical lié à sa précision topographique (Rainville et al., 2013).

Le taux de réalisation de la chirurgie était faible dans notre contexte car peu accessible à la population et elle n'est pas sans complications pour 
les patients âgés déjà très fragiles et sujettes à de multiples comorbidités. Le traitement a donc été médicamenteux dans la grande majorité des cas.

L’âge avancé de nos patients explique en partie la $2^{\text {ème }}$ position des lomboradiculalgies symptomatiques des tumeurs malignes devant les spondylodiscites contrairement aux résultats obtenus chez l'adulte jeune (Houzou et al., 2013). Le fort taux de cancer primitif indéterminé s'explique en partie dans notre étude par l'insuffisance du plateau technique pour un meilleur diagnostic étiologique des cancers. Seules les spondylodiscites infectieuses à $\mathrm{BK}$ probable ont été retrouvées dans notre étude. La prédominance des spondylodiscites infectieuses à $\mathrm{BK}$ dans la littérature (Cherif et al., 2013; Oniankitan et al., 2009) semblent se confirmer dans notre étude et sont avant tout le reflet du sous -développement et du manque d'hygiène.

\section{Conclusion}

La lomboradiculalgie commune est une pathologie fréquente de nos jours, tant dans la population togolaise que chez le sujet âgé en particulier. Il faut néanmoins s'acharner à éliminer une lomboradiculalgie secondaire chez le sujet âgé, dont le tableau clinique peut être trompeur et tenir aussi compte des pathologies associées à cet âge.

\section{References:}

1. Cecchi F, Debolini P, Lova RM, Macchi C, Bandinelli S, Bartali B et al. (2006). Epidemiology of back pain in a representative cohort of italian persons 65 years of age and older. The Inchianti Study. Spine; 31: 1149-55.

2. Cherif, I., Saidane, O., Mahmoud, I., Tekaya, R., Sahli, H., Abdelmoula L et al. (2013). Infectious spondylodiscitis in elderly patients: a report of 22 cas. BMJ ; 72 (3) : 1136.

3. Cheour, E., Hamdi, W., Tekaya R et al. (2007). Les lombosciatiques communes du sujet âgé : à propos de 67 cas. TunMéd ; 85 : 549-52.

4. Diomande, M., Eti, E., Ouali, B., Kouakou, E S., Outtara, M Y., \& Kouassi J M D et al. (2015). Profil des affections ostéoarticulaires des sujets âgés noirs africains : à propos de 157 cas vus à Abidjan. TunMéd; $93: 312-5$.

5. Haddada F, Ben HS, Younes M et al (2005). La sciatique commune du sujet âgé. Rev Rhum [Ed Fr];72: 1221(Abst; Me.76).

6. Houzou, P., Oniankitan, O., Kakpovi, K., Koffi-Tessio, V., Tagbor, KC., Fianyo et al. (2013). Profil des affections rhumatismales chez 13517 patients ouest africains.Tun Méd.; 91 : 16-20.

7. Institut de Recherche et Documentation en Economie de la Santé (2013). Politique de l'âge et santé. ADSP ; 85 : 20-5. 
8. Institut National de la Statistique et des Etudes Economiques et Démographiques (2015). Questionnaire unifié des indicateurs de base du bien-être. (Consulté le 05/11/2016). Disponible à partir de URL : <http://www.stat-togo.org/contenu/pdf/pb/pb-rap-finalQUIBB-tg-2015.pdf>.

9. Jiménez-Sánchez, S., Fernández-de-las-Peñas, C., Carrasco-Garrido, P., Hernández-Barrera, V., Alonso-Blanco, C., Palacios-Ceña D, et al. (2012). Prevalence of chronic head, neck and low back pain and associated factors in women residing in the autonomous region of Madrid (Spain). GacSanit; 26: 534-40.

10. Longue vie et autonomie. Le vieillissement humain (2016). Disponible à partir de URL: http: //www. longuevieetautonomie.fr /sites /default /files /editor /files /docs/1131088938792249. pdf.Consulté le 25/09/2016.

11. Mehrsa K, Björn R, Ralph H, Jan-Åke N et al. (2017). Risk factors for low back pain and sciatica in elderly men. The MrOS Sweden study. Age Ageing ; 46 : 64-71.

12. OMS (2016). Population mondiale. Disponible à partir de URL : http://www.populationmondiale.com/Consulté le 22 novembre 2016.

13. Ouédraogo DD, Bori-Bata F, Drabo YJ et al (2011). Sujet âgé et affections rhumatologiques en consultation de rhumatologie au Burkina Faso. Rev Rhum [Ed Fr];78: A957 (Abst; Me.149).

14. Oniankitan, O., Magnan, A., \& Fianyo, E. (2008). Lomboradiculalgie en consultation rhumatologique à Lomé (Togo). Rev Rhum ; 75: 3167.

15. Oniankitan, O., Bagayogo, Y., Fianyo, E., Koffi-Tessio, V., Kakpovi, K., Tagbor KC et al. (2009). Spondylodiscites infectieuses en milieu hospitalier à Lomé (TOGO). Med trop.; 69 : 581-2.

16. Rainville, J. \& Lopez, E. (2013). " Comparison of Radicular Symptoms Caused by Lumbar Disc Herniation and Lumbar Spinal Stenosis in the Elderly." Spine. 38(15):1282-1287.

17. Rahali-khachlouf, H., Hamdoun, S., Esseghir, S et al. (2009). Profil épidémio-clinique des lombosciatiques du sujet âgé. R F GérGéro ; $16: 270-6$.

18. Sghir, M., Zrour, S., Jellad, A., Ben, SZ., Salah S, Gharbi M et al (2011). Aspects récents de la sciatique commune du sujet agé. Rev Rhum [Ed Fr];78: 961(Abst; Lu.146).

19. Werndle, MC., Rega, A., \& Wrong, K. (2012). Acute disc herniation in the elderly. Br Jr Neuro Surg ; 26 (2) : 255-7.

20. Wong, AYL, Karppinen, J., \& Samartzis, D. (2017). Low back pain in older adults: risk factors, management options and futures directions. Scoliosis and Spinal Disorders ; 10 : 1186-96. 